\title{
LAS TECNOLOGÍAS DE REPRODUCCIÓN ASISTIDA Y SUS METÁFORAS
}

\author{
Ana Sánchez \\ Universidad de Valencia
}

ABSTRACT The enormous development -in both research and application- of assisted reproduction technologies has been accompanied by a lack of theoretical reflection on its power of change: the concept and treatment of the body, the concept of paternity or motherhood, etc.The metaphorical analysis allows us to deconstruct both dominant discourses: patriarchal and reductionist biomedical. We propose a transdisciplinary analysis which explains the interactions between the diverse discourses: biomedical, mass media, legal, symbolicpsycho-social, gender, historic- which make up the supply as well as the creation of demand of all these practices. The transdisciplinary analysis would help to reveal the biomedical interests and the acritical spirit of the mass media, and would supply a true evaluation of the results, risks, success, or viability, etc of these technologies.

KEY WORDS Metaphor. Bodies. Assisted reproduction.
RESUMEN El enorme desarrollo -tanto en la investigación cuanto en la implementación- de las tecnologias de reproducción asisiti$\mathrm{da}$, ha ido acompañado por una falta de reflexión teórica sobre su alcance transformador: la concepción y tratamiento de los cuerpos, las nociones de maternidad y paternidad, etc... El análisis metafórico posibilita la deconstrucción de los dos discursos dominantes en esta cuestión: el patriarcal y el biomédico reduccionista. Proponemos un análisis transdisciplinar que de cuenta de las interacciones entre los diversos discursos -biomédico, mediático, jurídico, simbólicopsico-social, de género, histórico- que conforman tanto la oferta como la generación de la demanda de estas prácticas. Que nos ayuden a desvelar los intereses biomédicos y el espiritu acrítico de los medios de comunicación, que nos posibilite una verdadera evaluación de los resultados, riesgos, éxitos, viabilidad, etc. de estas tecnologías.

PALABRAS CLAVE Metáfora. Cuerpos. Reproducción asistida.
Las tecnologías de reproducción asistida comportan un conjunto de aspectos teóricos, tecnológicos, sociológicos, juridicos, mediáticos, psico-simbólicos para cuya comprehensión se precisa un marco teórico complejo y transdisciplinar. Para la elaboración de este marco se ha recurrido a la epistemologia de la complejidad de Edgar Morin, a los estudios en Ciencia, Tecnologia y Sociedad y, en tercer lugar, a los análisis de género elaborados por la teoría feminista en relación al cuerpo de las mujeres y, más en concreto, a los temas de mujeres, maternidad y reproducción.

Partimos de considerar el carácter situado e histórico de la ciencia y el discurso científico. La ciencia, sus programas y formas de investigación pertenecen a una sociedad de cuyos valores generales se alimenta. Estos valores actúan como supuestos subyacentes -dificiles de identificar, por tanto. Los estudios en Ciencia, Tecnologia y Sociedad muestran: a) las imbricaciones que existen entre ciencia y tecnología (ya no se considera que una y otra pertenezcan a esferas separadas), y b) la inseparabilidad entre los aspectos sociológi- cos económicos, y lo especificamente teórico/tecnológico. Desde esta perspectiva, la ciencia deja de ser un conocimiento únicamente teórico para pasar a ser considerada en su acción, en sus consecuencias. En efecto, Jose Sanmartin presenta las tecnologías como el resultado de una elaboración teórica que después será utilizada en

- investigación científica;

- producción de artefactos (farmacia)

- tratamiento de los cuerpos (medicina)

- aplicaciones diversas (agricultura, etc.)

No olvidemos que la tecnología es intrinseca a los procesos de investigación, producción e implementación de las técnicas. Y este es el caso en las tecnologias de reproducción asistida, en las que las fronteras entre investigación e implementación son casi imperceptibles. No olvidemos que las tecnologias de reproducción asistida apenas han sido investigadas en animales, como paso previo a su aplicación en humanas. Tampoco olvidemos que en los mismos laborato- 
rios donde se realizan las fecundaciones in vitro, las criopreservaciones, se está investigando -con material genético cuya procedencia no se explicita- en nuevas técnicas y mejoras sofisticadas de las anteriores (pongamos como ejemplo el ICSI, esto es, inyección intracitoplasmática).

La tecnología está vinculada a la idea de progreso social: por ello, en el caso de las tecnologías de reproducción asistida se hace tan difícil la crítica social y/o mediática. Y también está vinculada a la economía y a diversas políticas (legislativas, institucionales, etc.). El ideal lineal que permea nuestra imagen del progreso científico-tecnológico

Ciencia-> tecnología-> industria-> mercado-> progreso

Debiera sustituirse por una modelización en forma de bucle que muestre la inseparabilidad y múltiples imbricaciones de estos aspectos

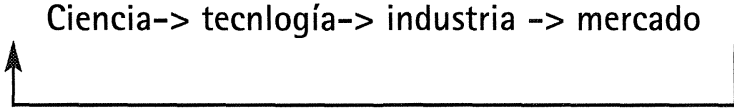

Por otra parte, la antigua idea -que sigue dominando entre la comunidad científica- que separa a la ciencia y la tecnologia de sus usos y consecuencias, sigue haciendo muy dificil la critica a los malos efectos de las tecnologías. La nueva corriente de Ciencia, Tecnologia y Sociedad ha propuesto la idea de evaluación de tecnologías para intentar analizar y subsanar estos efectos no deseados. La evaluación de tecnologías consiste en el conjunto de métodos que permiten analizar los impactos de una tecnología y valorarlos. Es decir, considerarlos desde sus impactos sociales, y hacer recomendaciones de uso. Estos serian los pasos: 1)identificación; 2) análisis; 3) valoración y 4) recomendaciones (véas más adelante).

El estudio de las tecnologías de reproducción asistida tendrá que considerar la interacción entre los aspectos

Sociales $->$ Económicos $->$ Politicos $->$ Científico-técnicos $\uparrow$

Y, por ello, los diferentes actores:

* Sociales: Mujeres (fundamentalmente) y hombres en tanto que objetos y sujetos de las tecnologías de reproducción asistida.

ARBOR CLXXXI 716 NOVIEMBRE-DICIEMBRE (2005) 523-530 ISSN: 0210-1963
* Económicos: industrias farmacéuticas; clínicas privadas y clínicas públicas, etc.

* Políticos: Aspectos jurídicos. Políticas estatales de reproducción

* Biomédicos

* Ideológicos y teóricos: discursos bioético, católico, feminista.

\section{El discurso biomédico}

En biomedicina perviven modelizaciones y conceptualizaciones de origen darwiniano. Se siguen utilizando como supuestos no revisados que sirven de guia heuristica para la investigación. Esto ocurre en las tecnologias de reproducción asistida. Sabemos que Darwin tomó de la selección artificial el modelo para elaborar su noción de selección natural. La selección artificial tiene varios rasgos: manipulación del cuerpo, control de los procesos reproductivos y fines estético/lucrativos que también están presentes en las tecnologías de reproducción asistida (se puede hacer selección de sexo, se venden -carísimos- ovulos de modelos, etc.). Además, estas metodologias pertenecen al paradigma biomédico reduccionista que fragmenta los cuerpos.

Para la identificación de tales supuestos subyacentes resulta muy útil el análisis de las metáforas en el lenguaje científico tal y como ha sido estudiado por Black, Hesse, Harding. El análisis metáforico: a) nos ayuda a desvelar e identificar tales supuestos, a ponerlos en cuestión y analizar las consecuencias que tienen en la concepción y prácticas biomédicas: la consideración del cuerpo, la elección de metodologias, etc.; y b) nos permite identificar las claves del sistema de creencias dominante dentro de un paradigma dado.

Remarquemos dos aspectos del análisis metafórico: a) revelan el sistema de valores y la forma de ordenamiento de una sociedad $y, b$ ) de forma recursiva, al nutrirse de determinados valores regulan y dirigen la acción investigadora también de determinada forma. Este carácter práxico de las metáforas tiene sus consecuencias en la metodología y diseño de Is programas de investigación, en la elección de lo que se considera problemático, es decir, en la decisión de seguir una investigación en detrimento de otras que se abandonan, así como en la valoración de los resultados (adelanto una pregunta: ¿vale la pena dedicar tanto dinero a una investigación cuya implementación tiene un $80 \%$ de fracasos?). 
Consideremos pues un doble juego metafórico que remite a dos discursos y a dos sistemas socio-simbólicos distintos aunque interrelacionados: el discurso científico y el discurso patriarcal: "el cuerpo es una máquina", "la mujer es madre». Ambas metáforas, con ondas raices en la historia de la ciencia occidental y del pensamiento occidental son de carácter ontológico (Lakoff y Jhonson). La primera, que entiende y modeliza al cuerpo humano como máquina, se relaciona con la metodología reduccionista y fragmentaria propia de la ciencia occidental antes aludida; la segunda, que fija a las mujeres con un único papel positivamente valorizado, el de madre, nos remite a claves antroposociales que no sólo son occidentales sino planetarios. Al considerar al cuerpo como máquina se descuida la mirada globalizadora y la indivisibilidad de la unidad psicosomática que acompaña tanto al deseo de maternidad, como a determinadas situaciones de "infertilidad». Al considerar a la mujer fundamentalmente como madre se eleva el deseo de maternidad a categoria determinante de la imprescindibilidad de estas investigaciones. En efecto, la oferta tecnológica en que se han convertido estas tecnologias se enmarcara tras un discurso que apela al deseo de maternidad (e incluso de paternidad). Así, en un bucle infernal la biomedicina justifica su oferta en la demanda de las mujeres, y, por ello mismo, las mujeres ya no pueden prescindir del recurso a esa oferta tecnológica. Tenemos que considerar las multiples raices enmarañadas (lingüisticas, lógicas, ideológicas y, todavia más profundamente, cerebro-psiquicas y socioculturales) que están en la base de la generación de la demanda de hijo junto con la respuesta de fragmentación y medicalización del cuerpo que da la biomedicina de las tecnologias de reproducción asistida. Como señala Silvia Tubert, al medicalizar la demanda de hijo se simplifica la cuestión, se pasa del orden simbólico -la demanda- a otro orden fenoménico real -la intervención.

La unión de las dos metáforas -cuerpo/fragmentación, mujer/madre- hace que en las intervenciones biomédicas el cuerpo de cada mujer se vaya reduciendo a vientre, útero, óvulos. Este proceso de objetivación hace que desaparezcan en tanto que sujetas. La práctica de la FIVTE (fecundación in vitro y transferencia de embriones), anula a la mujer concreta, reduce su cuerpo a unidades últimas e inconexas. Como tantas autoras han remarcado, en el proceso de medicalización de la maternidad, la esterilidad se ve convertida en enfermedad. Como nos dice Verena Stolcke, o en otros términos, Gena Corea, se resuelve un hecho complejo -con raices psicológicas, sociales y politicas- mediante una intervención biotécnica que, además, no cura la infertilidad.

\section{Otros discursos}

Se trata de entender que el orden sociosimbólico patriarcal permea y alimenta el discurso y prácticas biomédicas relativas a las tecnologias de reproducción asistida. También permea otros dos discurso relacionados con las tecnologías de reproducción asistida, el jurídico y el mediático; discursos que se entrealimentan con el biomédico y que, precisamente porque comparten las mismas claves sociosimbólicas patriarcales, no entran en contradicción, ni critican las prácticas biomédicas relativas a las tecnologías de reproducción asistida.

En efecto, y sobre todo en nuestro país, la prensa y la televisión informan de los adelantos y realizaciones de estas técnicas casi sistemáticamente de forma acritica. Lo muestran como grandes hallazgos, milagros tecnológicos que logran un fin deseado e incuestionable: la maternidad. Cabe suponer que con ello están haciendo difus ión de estos tratamientos y de la idoneidad de su aplicación. No informan de fracasos y riesgos, ni de las consecuencias físicas, económicas, personales de los embarazos múltiples (como el caso de Is sixtillizos de Huelva). También es de remarcar el cambio que ha habido en la publicitación mediática de estas tecnologias. En efecto, de una gran discreción inicial se ha pasado a publicitar los servicios, incluido el Diagnóstico genético preimplantacional tan discutido en otros países, 0 la compra de ovocitos/esperma.

\section{La teoría de los intereses en ciencia}

Pasemos a otro punto: los recientes estudios en Sociologia del Conocimiento Científico aportan otra interesante clave analitica: desvelemos, a través de las voces y discursos de los científicos tanto sus razones, cuanto sus intereses. Gena Corea dio en la clave de los intereses y móviles en muchos investigadores en tecnologías de reproducción asistida cuando reparó en un editorial escrito por el Doctor Alan Cherney en la revista Fertility and Sterility, ya en 1983, y en el que éste confesaba que los progresos tecnológicos en las tecnologias de reproducción asistida eran tan excitantes como haber sido físico en el Poyecto Manhattan. Por ello, lo denominó Gena Corea el "Proyecto Manhattan de Reproducción»-pues, en su opi- 
nión, tanto la bomba atómica como la fertilización in vitro ofrecen una solución de alta tecnología a un problema que precisa de soluciones politicas y sociales (y en nuestro caso también psicológicas). Una de las características que comparten ambos programas de investigación son las pugnas y prisas que se detectan entre diversas equipos investigadores. La carrera en las tecnologias de reproducción asistida, lo sabemos, ha sido vertiginosa:

1978: nace Louise Brown, la primera «niña probeta».

1979: ya se utiliza la donación de esperma -se fertiliza a una mujer con esperma que no es de su compañero.

1982: comienzan las investigaciones sobre donación de óvulos -así una mujer puede albergar embriones no engendrados por ella.

1983: primera FIV con ovulo donado en Australia.

1984: primer nacimiento a partir de un embrión congelado en Australia.

A partir de ahí, avanzan las investigaciones sobre cribado de embriones - posibilidad de selección de rasgos, sexo, posibilidad de desechar embriones defectuosos; se sofistican los procedimientos FIV, se investiga en infertilidad masculina "solucionándose" con el procedimiento ICSI.

Desde la perspectiva amplia que entrelaza los diversos aspectos del desarrollo de cualquier tecnología tenemos que considerar que las tecnologias de reproducción asistida se han convertido para médicos, biólogos, farmaceúticos en un punto de investigación fundamental. El incremento de las enormes inversiones dedicadas a este programa de investigación va unido a su espectacular desarrollo a) cuantitativo: multiplicación de número de centros especializados en investigación e implementación de las tecnologías de reproducción asistida; y b) cualitativo: renovación de las ofertas médicas.

\section{Los "hechos"}

Las tecnologias de reproducción asistida se presentaron como una solución médica a los problemas de infertilidad en mujeres debido a causas concretísimas: la obstrucción de trompas. En sus 20 años de existencia las tecnologias y aplicaciones se han extendido muchísimo. Veamos en primer lugar las aplicaciones: De aquella identificabe ano-

ARBOR CLXXXI 716 NOVIEMBRE-DICIEMBRE (2005) 523-530 ISSN: 0210-1963 malia, se ha pasado a considerar aconsejable aplicar estas técnicas en muchos casos de "infertilidad" que no están tan claros. La misma noción de infertilidad ha ido remodelándose. Veamos una clásica definición de infertilidad para la que se considera aconsejable el tratamiento con tecnologías de reproducción asistida: la infertilidad -sea ésta masculina o femenina se define como la imposibilidad de una pareja para lograr la concepción o llevar un embarazo hasta su término, al menos después un periodo de un año de relaciones sexuales normales y sin protección. En esta definición se pasa a considerar la adecuación de tratamiento aún en los casos en que se desconoce la causa. Es cierto que hoy dia se reconoce la existencia de la infertilidad masculina, pero curiosamente, cuando está claramente identificada, ésta se "trata" en el cuerpo de la mujer con el procedimiento ICSI. Las causas objetivas de infertilidad femenina son trastornos de la ovulación y anomalias en las trompas. También, la endometriosis y la hiperprolactina. Las causas objetivas de infertilidad masculina son la oligospermia (escaso número de espermatozoides), la teratospermia (anomalias diversas) y la astenospermia (baja movilidad). Pero existe una banda intermedia de esterilidad no explicada, o de causas de esterilidad masculina que tienen que ver con factores ambientales alimentación, exceso de calor en el medio laboral, etc., y cuya solución se tecnologiza con el recurso a las tecnologias de reproducción asistida.

\section{La metáfora de la culpa y la asimetría en los tratamientos}

En un tratamiento FIVTE se suelen seguir los siguientes pasos:

1) Estimulación del ovario con hormonas

2) Recogida de ovocitos (mujer) y de esperma (hombre)

3) Fase in vitro: Inseminación de los ovocitos en el laboratorio

4) Cultivo in vitro del ovulo fecundado hasta que el embrión tenga entre 2 y 8 células ( $48-72$ horas)

5) Transplante embrionario

Veamos ahora, en un cuadro esquemático, qué supone un ciclo FIV que funcione sin problemas para el cuerpo de la mujer (y qué supone para el del hombre). Al analizar el cuadro nos detendremos en tres momentos especialmente dificiles: 


\begin{tabular}{|c|c|c|c|}
\hline & \multicolumn{2}{|l|}{ Mujer } & Varón \\
\hline $1{ }^{\circ}$ Mayo & \multicolumn{2}{|c|}{ Menstruación (o interrupción de la píldora, si es un ciclo programado) } & \\
\hline 2 al 11 Mayo & \multicolumn{2}{|c|}{$\begin{array}{l}\text { Tratamiento de estimulación ovárica con FSHr. Maduración de varios } \\
\text { foliculos }\end{array}$} & Espermocultivo \\
\hline \multirow[t]{2}{*}{ 10/11 Mayo } & \multirow[b]{2}{*}{ Control } & $\begin{array}{l}\text { Ecografía ovárica: } \\
\text { Balance de foliculos. }\end{array}$ & \\
\hline & & $\begin{array}{l}\text { Dosificación de estradiol en la sangre: } \\
\text { Estimación de la madurez de los foliculos }\end{array}$ & \\
\hline 11 Mayo & \multicolumn{2}{|c|}{ Desencadenamiento de la ovulación por hCG } & \\
\hline 13 Mayo & $\begin{array}{l}\text { Recogida de } \\
\text { óvulos }\end{array}$ & $\begin{array}{l}\text { Celioscopia: anestesia general o local } \\
0 \\
\text { Punción bajo control ecográfico }\end{array}$ & $\begin{array}{l}\text { Recogida } \\
\text { de } \\
\text { esperma }\end{array}$ \\
\hline 13 al 15 Mayo & Fase in vitro & $\begin{array}{l}\text { Puesta en cultivo de los óvulos } \\
\text { Recogida y tratamiento del esperma } \\
\text { Fecundación y cultivo del embrión }\end{array}$ & \\
\hline 15 Mayo & \multicolumn{2}{|c|}{ Transplante embrionario : 1 a 3 embriones en el útero } & \\
\hline \multirow[t]{2}{*}{18 al 27 Mayo } & \multicolumn{2}{|c|}{ Control cotidiano de la temperatura } & \\
\hline & $\begin{array}{l}\text { Controles } \\
\text { Hormonales } \\
\end{array}$ & $\begin{array}{l}\text { Calidad de la secreción ovárica } \\
\text { (Estradiol y progesterona) } \\
\text { Detección de la hormona embrionaria }\end{array}$ & \\
\hline 27 Mayo & \multicolumn{2}{|r|}{${ }_{0}$ Comienzo del embarazo } & \\
\hline
\end{tabular}

1. El tratamiento con diversas hormonas durante todo el ciclo.

Para incitar a una mayor producción de folículos se administra clomifeno junto con gonadotropina (HMG: asociación de FSH -hormona de estimulación folicular-y LH -homona luteinizante). Unos dias después se desencadenará la ovulación mediante HGC, es decir gonadotropina coriónica humana, que completa la maduración de los ovocitos existentes en el interior de los folículos.

2. La «recogida» de óvulos, es decir, su extracción, que se efectúa por punción bajo control ecográfico. Hay que entender que se extraen diversos ovocitos pero en distintos grados de maduración. La punción de ovarios para la extracción de los óvulos pueden ocasionar hemorragias, infecciones, e incluso perforaciones.

3. Transplante embrionario. Posible congelación de embriones: habituamente se transfieren unos tres embriones; los restantes se congelan y pueden ser utilizados en caso de que el embarazo que se espera en la fase 5 falle, o también si se desea un nuevo hijo.

En la literatura especializada (veasé, por ejemplo el artículo de Ana Marti) se remarca el estado de ansiedad y los trastornos físicos que las mujeres suelen experimentar como consecuencia de los tratamients hormonales. También aumenta su ansiedad en los dias posteriores a la transferencia. Dado que se establece un tiempo de espera para constatar el éxito del embarazo. Como sabemos, los riesgos de rechazo son altísimos: recordemos el escaso índice de éxitos.

He intentado mostrar en la tabla anterior la asimetría entre el tratamiento que recibe el cuerpo de la mujer y el del hombre. Parece "colarse" aqui para los investigadores/as e implementadores/as aquella otra metáfora que echa la culpa de todo a la mujer. Un caso muy especial en mi opinión consiste en la investigación para resolver la infertilidad masculina que se han centrado en la inyección intracitoplasmática (ICSI): ésta consiste en la microinyección de un espermatozoide en un sólo ovocito. Hay que seguir casi todas las fases FIV. Es decir, que el tratamiento se hace fundamentalmente en el cuerpo de una mujer que sí es fértil. El espermatozoide se puede obtener del eyaculado, pero también del epidímio o del testículo mediante una biopsia. En 1977, Françoise Laborie ya nos hizo reflexionar: en Francia, donde ha habido más debate sobre los riesgos y la ideoneidad de seguir o abandonar alguna de estas técnicas, prácticamente nadie, a excepción del discurso feminista, se habia preocupado por los riesgos de aplicación de la FIVTE; sin embargo, en el caso de la ICSI, biólogos, genetistas, médicos piden más experimentación animal y cierta demo- 
ra en la aplicación real. Aqui si se reflexiona sobre riesgos para la descendencia, quirúrgicos y se alude a falta de conocimientos sobre procesos fundamentales.

Donación de ovocitos: la mujer constituye, evidentemente, lo que en biología se denomina un "recurso limitado». Su producción de gametos es escasa, y éstos constituyen, al mismo tiempo, el material básico donde realizar las investigaciones. Tanto en la recaptación de ovocitos como en la criopreservación de embriones se observa el interés biomédico para disponer de material destinado a investigaciones que trascienden los intereses de la reproducción. Hasta ahora se había querido separar el estudio e implementación en tecnologías de reproducción asisitida de investigaciones en clonación y en células madre. En mi opinión, están profundamente imbricadas, dado que para estas últimas investigaciones se precisa el material sobrante que las tecnologias de reproducción asisitida pueden proporcionar. Esa debe ser la razón de que los y las biomédicos estén exigiendo la libre utilización de los más de 200.000 embriones que hoy dia hay preservados sólo en nuestro pais. Por otra parte, las investigaciones en células madre podrian realizarse a partir de otros tejidos humanos, como la médula ósea, menos problemáticos desde el punto de vista ético, legal, y sobre todo de utilización de recursos humanos, que en el caso de los embriones.

\section{Evaluación de riesgos y resultados}

Diversos investigadores/implementadores de las tecnicas de reproducción asistida están de acuerdo que para valorarlas hay que atender a "una triple perspectiva de eficacia, coste y riesgo" (Barri et al., p. 149). La cuestión es cómo analizar tales variables. No hay una verdadera evaluación de los riesgos. Se reconoce las posibles "complicaciones": a saber, embarazo múltiple, hiperestimulación ovárica, cáncer ginecológico. Desglosemos brevemente estos tres aspectos:

La eficacia, la extraña forma de mostrar/ocultar los datos. Las tecnologías de reproducción asistida no solucionan el gran salto que hay entre embarazo e hijo a término. Mientras que un $71 \%$ de fertilizaciones naturales tienen como resultado el nacimiento de una criatura, en la FIV es cuanto más un $20 \%$. Este bajo indice aumenta en función del número de intentos. Curiosamente, los datos que dan entidades investigadoras de gran renombre en nuestro pais se refieren siempre a los embarazos. Ello es engañoso pues se puede confundir con hijos a término. Preguntados porqué no ofrecen estadísticas sobre los hijos nacidos llegan a esgrimir argumentos tan poco científicos como que no pueden hacer seguimientos ya que las parejas desaparecen tras el tratamiento. Es bien dudoso, pues al mismo tiempo las consultas se ven repletas de fotos de las criaturas nacidas y cuyos padres desde luego han agradecido a la entidad enviando la foto. Tendriamos que suponer que la uno información» (es decir, que los padres no informen a las clínicas del nacimiento) en realidad es información pero de datos negativos.

Los costes: es importante distinguir entre las clínicas públicas y las privadas pòr su diferentes estatus de disponibilidad económica, y por tanto capacidad de comprar óvulos, y otros tipos de recursos. Es curioso que no existan análisis económicos de las tecnologías de reproducción asistida; análisis que debieran atender un doble aspecto:

a) que los tratamientos en general son muy costosos -a lo que se añade el elevado número de fracasos (un 70$80 \%$ ). Es decir, que en las clínicas privadas te cobran elevadas tarifas sin garantizarte el éxito del tratamiento; y

b) que las hormonas utilizadas en el proceso de estimulación también son muy costosas. Por ello debieran realizarse análisis y control de las industrias farmaceúticas. No podemos olvidar que los más importantes laboratorios que elaboran las hormonas utilizadas en un ciclo FIVTE patrocinan al mismo tiempo publicaciones, encuentros y congresos en nuestro pais: los laboratorios Organon, que producen Puregon (FSH recombinante) publica las "Actualizaciones de la Sociedad Española de Fertilidad 2000"; el grupo Menarini edita el VIII curso intensivo de formación continuada"; y, los laboratorios Serono, "Novedades Terapeúticas sobre Reproducción Humana", por poner unos ejemplos. En estas publicaciones encontramos los nombres, institutos y clínicas privadas más prestigiosos de España.

Los riesgos: sin embargo, las críticas arriba apuntadas acerca del modelo de ciencia y la implementación concreta de estas tecnologías en el cuerpo de las mujeres, nos lleva ahora a identificar los riesgos que les acompañan, asi como a considerar que las llamadas "complicaciones" en los tratamientos constituyen en realidad graves problemas, insosla- 
yables de momento. El problema reside en identificar qué cosas perciben los biomédicos como riesgos. He podido observar que casi nunca se reconocen los riesgos, sólo se mencionan indirectamente cuando se presenta una nueva técnica que resuelve un problema anterior. Es decir, sólo a posteriori cuando se ha encontrado una alternativa, se reconoce que en la metodología anterior había riesgo. López Cerezo y Luján reclaman políticas para «reducir riesgos, y atribuir responsabilidades por omisión, o bien pueden diseñarse políticas para remediar daños ya producidos» (pág. 139). Es cierto que las mujeres asumen el riesgo, pero es de remarcar que no se piden moratorias de ningún tipo en la implementación de estas tecnologías. Resumiremos, muy brevemente, algunas de las críticas hechas a la rapidez con que se aplican a los cuerpos de las mujeres las diversas técnicas, es decir, la casi ausencia de estudios longitudinales. Además, como comenta la Doctora Paloma Gómez, incluso las pruebas diagnósticas tienen sus riesgos: la histerosalpingografía -se introduce un líquido de contraste en útero y trompas para explorar la permeabilidad-puede dar lugar a cuadros de peritonitis aguda. Por otra parte, el tratamiento hormonal-que inducirá la estimulación ovárica que se realiza en todos los ciclos FIV- puede desarrollar un síndrome de hiperestimulación ovárica (agrandamiento quístico de los ovarios), presencia de ascitis o incluso de derrame pleural . Por otra parte, no existen estudios sobre las consecuencias que pueda tener la estimulación ovárica en un posible adelanto de la menopausia.

\section{¿Conclusiones?}

El análisis de las tecnologias de reproducción asistida requiere pues un enfoque plural que refleje los distintos discursos de poder y las distintas voces -en tanto que sujetas o sujetos epistémicos- que participan en esta cuestión. Los discursos legitimadores son el biomédico y el jurídico. Las mujeres -fundamentalmente- y también los varones que se someten a las técnicas de reproducción asistida constituyen los sujetos (al mismo tiempo que objetos). Hay también otras voces: las feministas, la iglesia católica. Los discursos subyacentes los identificamos en todas las construcciones psico-sociales de roles e identidades de sexo/género en mujeres y varones tanto desde la estructura social cuanto desde el imaginario colectivo. Las tecnologias de reproducción asistida apenas han encontrado discursos opositores -a excepción del feminista y el-de la iglesia. Los medios de comunicación las encuentran fascinantes. Se da una per- fecta sintonia entre discurso biomédico, discurso mediático y expectativas sociales.

Una perspectiva planetaria nos haria aumentar las dudas sobre la idoneidad de estos tratamientos. Los seis mil millones de habitantes de nuestro planeta no parecen apuntar a la necesidad de investigaciones sobre fertilización in vitro. Es decir, la apuesta médica se invierte: en el norte, búsqueda impenitente de la maternidad (genética), en los sures de todos los sures, planificación y control de la misma.

Una última palabra sobre las metáforas: la metáfora cubre ese interfaz entre lo simbólico y lo real. La tradición analítica en filosofía de la ciencia siempre ha desdeñado sus aspectos simbólicos. Sin embargo, entre la realidad y nuestras interpretaciones se alza un velo de proyecciones/identificaciones. En la tradición cientifica se ha intentado expulsar lo simbólico cuando una de las principales caracteristicas de homo sapiens es su capacidad de ritualizar. Es preciso por tanto, no soslayar los aspectos simbólicos: la forma de interpretación/mediación con la realidad que es peculiar en homo sapiens. Asi, de las dos metáforas iniciales: el cuerpo como máquina y la mujer como madre, que pertenecen a dos órdenes sociosimbólicos que se entrecruzan y entrealimentan, el biomédico y el patriarcal, tenemos que considerar que el desarrollo investigador de las tecnologias de reproducción asistida responde al primer orden pero lleva implícito el segundo. En esta separación entre el discurso biomédico dominante (con la consecuente medicalización de la maternidad y la esterilidad) y los ordenamientos psico/socio/culturales que generan la demanda es lo que soslaya el análisis y probable búsqueda de soluciones en los entramados de este segundo aspecto. Por ello se da una respuesta biotécnica a una cuestión que: a) ha sido convertida en tremendo problema, b) tiene múltiples causas y c) por todo ello, a su vez, puede tener muy diversas soluciones.

Y acabo, como es mi gusto, con una pregunta: ¿por qué se someten las mujeres a técnicas dolorosas, perjudiciales y costosas, con escaso indice de éxito? Una de las respuestas podría proceder de análisis etnometodológicos en los que pudieran emergen las voces de las mujeres, y desde luego también las de los varones. Hemos tomado las metáforas como desveladoras del sistema de creencias general. Ello puede ayudarnos a entender qué metodologias se utilizan y qué programas de investigación se prefieren. Es decir, cuáles de ellos son punteros. ¡Buena metáfora! 


\section{BIBLIOGRAFÍA}

Actualizaciones de la Sociedad Española de Fertilidad. SEF-Organon

Akrich, Madeleine y FranÇoise Laborie. De la contraception à l'enfantement. Cahiers du genre. Ed. L'Harmattan, 1999

Barri (et al.) Novedades Terapéuticas sobre Reproducción Humana. Excerpta Medica, 2000.

Black, Max, Models and metaphors, Ithaca, NY, Cornel University Press, 1962. Trad. esp., Modelos y metáforas, Madrid, Tecnos, 1966.

Cabero (et al.) VIII curso intensivo de formación continuada. Endocrinologia ginecológica y reproducción humana. Eds.Ergon, 2000

Comisión Nacional de Reproducción Asistida. I Informe Anual, 1998

Corea, Gena. The Mother Machine. Harper and Row, N.Y. USA, 1985

Gómez, Paloma. "Reflexiones de una médica de familian. Texto inédito. En el Curso sobre Técnicas de reproducción asistida: problemática médica y social, UIMP, 1999, Valencia

Harding, Susan. Ciencia y feminismo. Ed. Morata, Madrid, 1996

Hesse, M., Models and Analogies in Science, Notre Dame, IN, University of Notre Dame Press, 1966.

Laborie, FranÇoise. "Procreation artificielle: santé des femmes et des enfants", en Santé et mortalité des enfants en Europe. UCL/ULB, I'Harmattan, 1996

LakofF, G. y Johnson, M. Metaphors we live by, Chicago, University of Chicago Press, 1980.Trad. esp. Metáforas de la vida cotiddiana, Madrid, Cátedra, 1986.

López Cerezo, José A. y Luján, José Luis. Ciencia y Política del riesgo. Alianza Ed., Madrid, 2000

Luján, Jose Luis. "El estudio social de la tecnologian, en Estudios sobre sociedad y tecnologia, Sanmartin (et al.) eds., Anthropos, 1992

Marti, Ana. "Dilemas en torno a la maternidad. Experiencias de mujeres", Texto inédito. En el Curso sobre Técnicas de Reproducción asisitida: problemática médica y social. UIMP de Valencia, 1999

Morin, Edgar, El Método, vol. IV, Las ideas. Cátedra, 1992.
Sanmartin, José y 0. rti, Angel, "Evaluación de tecnologias", en Estudios sobre sociedad y tecnología, Sanmartin (et al.) eds., Anthropos, 1992

Testart, Jacques. El embrión transparente, Granica, Barcelona, 1988.

Tubert, Silvia. Mujeres sin sombra. Maternidad y tecnología. Madrid, S.XXI, 1991.

ARBOR CLXXXI 716 NOVIEMBRE-DICIEMBRE (2005) 523-530 ISSN: 0210-1963 